\title{
NONINCLUSION THEOREMS FOR SUMMABILITY MATRICES
}

\author{
J. A. FRIDY \\ Department of Mathematics and Computer Science \\ Kent State University \\ Kent, Ohio 44242
}

(Received November 30, 1995)

\begin{abstract}
For both ordinary convergence and $\ell^{1}$-summability explicit sufficient conditions on a matrix have long been known that ensure that the summability method is strictly stronger than the identity map The main results herein show that a matrix that satisfies those conditions can be included by another matrix only if the other matrix satisfies those same conditions.
\end{abstract}

KEY WORDS AND PHRASES: regular matrix, $\ell-\ell$ matrix, (summability) inclusion, SilvermanTöeplitz conditions, Knopp-Lorentz conditions.

1991 AMS SUBJECT CLASSIFICATION CODES: 40D25, 40C05

\section{INTRODUCTION AND TERMINOLOGY}

Let $x$ denote a complex number sequence $\left\{x_{k}\right\}_{k=1}^{\infty}$ and $A$ denote an infinite matrix $\left[a_{n k}\right]$ with complex entries; then $A x$ is the transformed sequence whose $n$-th term is given by $(A x)_{n}=\sum_{k=1}^{\infty} a_{n k} x_{k}$. Let $c$ denote the set of convergent sequences, and $c_{A}=\{x: A x \in c\}$ Similarly, $\ell^{1}=\left\{x: \sum_{k=1}^{\infty}\left|x_{k}\right|<\infty\right\}$ and $\ell_{A}=\left\{x: A x \in \ell^{1}\right\} \quad$ The matrix $A$ is called regular if $c \subseteq c_{A}$, and $A$ is stronger than (ordinary) convergence if $c \stackrel{c}{\neq} c_{A}$ Similarly, $A$ is called an $\ell-\ell$ matrix if $\ell^{1} \subseteq \ell_{A}$, and $A$ is stronger than the identity (map) if $\ell^{1} \underset{\neq}{\subset} \ell_{A}$. If $A$ and $B$ are matrices such that $\lim A x=L$ implies $\lim B x=L$, then we say " $B$ includes $A$," and this clearly implies that $c_{A} \subseteq c_{B}$. In the $\ell-\ell$ case we simply write $\ell_{A} \subseteq \ell_{B}$ with no verbal phase describing it.

There is previous work giving explicit conditions on $A$ to imply that $c_{A}=c=c_{I}$ or $\ell_{A}=\ell^{1}=\ell_{I}$ (See, e.g., the Mercerian-type theorems in [1], [3], and [4]. In [2] and [5] conditions on $A$ were given that ensure that $c \underset{\neq}{\subset} c_{A}$ and $\ell^{1} \underset{\neq}{\subset} \ell_{A}$, respectively Explicit conditions are not known for making general comparisons of $c_{A}$ and $c_{B}$ or of $\ell_{A}$ and $\ell_{B}$ (except when $B=I$ ) In this paper we address the general inclusion question. The principal results show that if $A$ satisfies the conditions of [2] or [5] that ensure that $A$ is stronger than $I$, then $A$ can be included by $B$ only if $B$ also satisfies those same conditions

For the reader's convenience we state the theorems due to Silverman-Toeplitz [6, page 43] and Knopp-Lorentz [7] that characterize regular matrices and $\ell-\ell$ matrices, respectively

SILVERMAN-TÖEPLITZ THEOREM. The matrix $A$ is regular if and only if the following conditions are satisfied:

(i) for each $k, \lim _{n} a_{n k}=0$,

(ii) for each $n, \sum_{k=1}^{\infty} a_{n k}$ converges and $\lim _{n} \sum_{k=1}^{\infty} a_{n k}=1$,

(iii) $\sup _{n} \sum_{k=1}^{\infty}\left|a_{n k}\right|<\infty$ 
KNOPP-LORENTZ THEOREM. The matrix $A$ is an $\ell-\ell$ matrix if and only if the following condition is satisfied:

(iv) $\sup _{k} \sum_{n=1}^{\infty}\left|a_{n k}\right|<\infty$.

\section{COMPARISON OF REGULAR MATRICES}

In [2] Agnew proved the following simple criterion for establishing $c \stackrel{c}{f} c_{A}$

THEOREM 2.1. If $A$ is regular and satisfies the condition

$$
\lim _{n, k} a_{n k}=0 \text {, }
$$

then $c \stackrel{c}{\neq} c_{A}$.

The double limit in (2.1) is taken in the Pringsheim sense: if $\epsilon>0$ then there exists an $N$ such that $\left|a_{n k}\right|<\epsilon$ whenever both $n>N$ and $k>N$. This sets the stage for the first of our "noninclusion theorems."

THEOREM 2.2. If $A$ and $B$ are regular matrices such that $A$ satisfies (2.1) and $B$ does not, i e.,

$$
\lim _{n, k} b_{n k} \neq 0,
$$

then $c_{A} \nsubseteq c_{B}$

PROOF. First note that since the rows of $A$ are null sequences, (2.1) implies that

$$
\lim _{k}\left\{\max _{n}\left|a_{n k}\right|\right\}=0
$$

Also, (2.2) allows us to choose increasing sequences $\nu^{\prime}$ and $\kappa^{\prime}$ of row and column indices satisfying

$$
\left|b_{\nu^{\prime}(m), \kappa^{\prime}(m)}\right| \geq \delta>0 \text { for all } m \text {. }
$$

Then use (2.3) to choose a subsequence of those pairs $\left\langle\nu^{\prime \prime}(m), \kappa^{\prime \prime}(m)\right\rangle$ such that

$$
\max _{n}\left|a_{n, \kappa^{\prime \prime}(m)}\right|<2^{-m} \text { for all } m \text {. }
$$

Next, using conditions ST(i) we choose a further subsequence $\langle\nu(m), \kappa(m)\rangle$ so that for each $m$, $k<\kappa(m)$ and $n>\nu(m)$ imply

$$
\left|a_{n k}\right|<2^{-m} \text { and }\left|b_{n k}\right|<2-m .
$$

We also use the assumption that the rows of $B$ tend to zero (from ST(ii)) to choose $\langle\nu(m), \kappa(m)\rangle$ so that

$$
\left|b_{n k}\right|<2^{-m}
$$

whenever $k>\kappa(m)$ and $n<\nu(m)$. Define the sequence $x$ by

$$
x_{k}:=\left\{\begin{array}{cl}
m, & \text { if } k=\kappa(m) \text { for } m=1,2, \ldots, \\
0, & \text { otherwise. }
\end{array}\right.
$$

For $n>\nu(m),(2.5),(2.6)$, and (2.7) yield

$$
\begin{aligned}
\left|(A x)_{n}\right| & =\left|\sum_{\jmath=0}^{\infty} a_{n, \kappa(\jmath)}(j)\right| \\
& \leq \sum_{\jmath \leq m} 2^{-m}(j)+\sum_{\jmath>m} 2^{-\jmath}(\jmath) \\
& \leq 2^{-m} \sum_{\jmath \leq m} j+R_{m},
\end{aligned}
$$


say As $m \rightarrow \infty$ these expressions both tend to zero $\left(R_{m} \rightarrow 0\right.$ because the series $\sum 2^{-\jmath} \mathrm{J}$ is convergent); hence, $\lim A x=0$ and $x \in c_{A}$. For $B x$ we have

$$
\begin{aligned}
\left|(B x)_{\nu(m)}\right| & \geq\left|b_{\nu(m), \kappa(m)} x_{\kappa(m)}\right|-\sum_{j \neq m}\left|b_{\nu(m), \kappa(\jmath)}\right|(j) \\
& =\left|b_{\nu(m), \kappa(m)} m\right|-\sum_{\jmath<m} 2^{-m}(j)-\sum_{\jmath>m} 2^{-\jmath}(j) \\
& =\left|b_{\nu(m), \kappa(m)}\right| m-2^{-m-1} m(m+1)-R_{m} .
\end{aligned}
$$

The latter two terms tend to zero as above, and by (2.4) the first term is unbounded, hence, $x \notin c_{B}$, and the proof is complete.

REMARK. In the proof of Theorem 2.2 we did not use the full strength of the regularity hypothesis. It would have sufficed to assume only that the rows and columns of $A$ and $B$ tend to zero.

To illustrate Theorem 2.2 we can take $A$ to be any Cesàro matrix $C_{\text {j }}$ for $j>0$, or any Euler-Knopp matrix $E_{r}$ for $0<r<1$. (They all satisfy (2.1).) Then $B$ could be any Norlund matrix $N_{p}$ with $p$ finitely nonzero (see [6, page 64]), or any weighted mean $\bar{N}_{p}$ with $p \in \ell^{1}$ (see [6, page 57]), they satisfy (2.2). Therefore none of the latter matrices includes any of the former.

One might note the similarity of form between Theorem 2.2 and Theorem 2.0.3 of [8] where Wilansky proved that if $A$ is conull and $\mathrm{B}$ is not, then $c_{A} \nsubseteq c_{B}$. The conservative matrix $A$ is conull provided that

$$
\lim _{n} \sum_{k=1}^{\infty} a_{n k}-\sum_{k=1}^{\infty}\left(\lim _{n} a_{n k}\right)=0
$$

\section{COMPARISON OF $\ell-\ell$ MATRICES}

In [5] the following theorem was proved, giving a sufficient condition for an $\ell-\ell$ matrix to be stronger than the identity matrix

THEOREM 3.1. If $A$ is an $\ell-\ell$ matrix for which there exists an integer $m$ such that

$$
\lim _{k} \inf \sum_{n=m}^{\infty}\left|a_{n k}\right|=0
$$

then $\ell^{1} \stackrel{c}{\neq} \ell_{A}$

We next give an $\ell-\ell$ analogue of Theorem 2.2 .

THEOREM 3.2. If $A$ and $B$ are $\ell-\ell$ matrices such that $A$ satisfies (3.1) and $B$ does not, then $\ell_{A} \nsubseteq \ell_{B}$

Actually, we shall prove somewhat more.

THEOREM 3.3. Let $A$ be an $\ell-\ell$ matrix for which there is an integer $\mu$ and a sequence $\{k(\jmath)\}_{\jmath=1}^{\infty}$ of column indices such that

$$
\lim _{\jmath} \sum_{n=\mu}^{\infty}\left|a_{n, k(\jmath)}\right|=0
$$

if $B$ is a matrix satisfying

$$
\lim _{j} \sum_{n=\mu}^{\infty}\left|b_{n, k(j)}\right| \neq 0
$$

then $\ell_{A} \nsubseteq \ell_{B}$.

PROOF. First note that we may assume that the rows of $B$ satisfy

$$
\lim _{\jmath} b_{n, k(\jmath)}=0 \text { for each } n \text {. }
$$


For, if not, then there is an $n^{*}$ and a subsequence $\left\{k^{\prime}(j)\right\}$ such that

$$
\left|b_{n^{\bullet}, k^{\prime}(\jmath)}\right| \geq \epsilon>0 \text { for every } j .
$$

Property (3.2) allows us to choose a further subsequence $\left\{k^{\prime \prime}(j)\right\}$ such that

$$
\sum_{n=\mu}^{\infty}\left|a_{n, k^{\prime \prime}(\jmath)}\right|<\frac{1}{j^{2}} \text { for each } j .
$$

Define

$$
x_{k}:= \begin{cases}1, & \text { if } k=k^{\prime \prime}(j) \text { for } \jmath=1,2, \ldots \\ 0, & \text { otherwise }\end{cases}
$$

This yields

$$
\begin{aligned}
\sum_{n \geq \mu}\left|(A x)_{n}\right| & =\sum_{n=\mu}^{\infty}\left|\sum_{\jmath=1}^{\infty} a_{n, k^{\prime \prime}(\jmath)}\right| \\
& \leq \sum_{\jmath=1}^{\infty} \sum_{n=\mu}^{\infty}\left|a_{n, k^{\prime \prime}(\jmath)}\right| \\
& \leq \sum_{j=1}^{\infty} \frac{1}{j^{2}}
\end{aligned}
$$

while (3.5) implies that the series

$$
(B x)_{n^{*}}=\sum_{\jmath=1}^{\infty} b_{n^{*}, k^{\prime \prime}(\jmath)}
$$

is nonconvergent. Thus, as in the proof of Theorem 1 of [5], we can choose $x$ so that $x \in \ell_{A}$ but $B x$ is not defined.

Assume that (3.2), (3.3), and (3.4) hold We shall find an $x$ in $\ell_{A}$ that is not in $\ell_{B}$. Using (3 3) and replacing $\{k(j)\}$ with one of its (appropriately chosen) subsequences $\{k(i)\}$, we can assume without loss of generality that

$$
\sum_{n=1}^{\infty}\left|b_{n, k(2)}\right| \geq 2 \delta>0 \text { for each } i
$$

Replacing $\{k(i)\}$ with yet another of its subsequences $\{k(p)\}$ we can get .

$$
\sum_{n=\mu}^{\infty}\left|a_{n, k(p)}\right|<t_{p} \quad \text { for each } p
$$

where $t \in \ell^{1}$.

Next we construct an increasing sequence $\{\nu(m)\}$ of row indices and a further subsequence $\{\kappa(m)\}$ of $\{k(p)\}$ to define the sequence $x$ that we seek First, take $\nu(-1)=0$; then use (36) to choose $\kappa(1)$ satisfying

$$
\sum_{n=1}^{\infty}\left|b_{n, \kappa(1)}\right| \geq 2 \delta
$$

and choose $\nu(1)$ so that

$$
\sum_{n \leq \nu(1)}\left|b_{n, \kappa(1)}\right| \geq \delta \quad \text { and } \sum_{n>\nu(1)}\left|b_{n, \kappa(1)}\right|<t_{1} .
$$

After $\kappa(1)<\ldots<\kappa(m-1)$ and $\nu(1)<\ldots<\nu(m-1)$ have been selected use (3 4) to choose $\kappa(m)>\kappa(m-1)$ such that 


$$
\sum_{n=1}^{\nu(m-1)}\left|b_{n, \kappa(m)}\right|<t_{m}
$$

and by (3.6),

$$
\sum_{n=1}^{\infty}\left|b_{n, \kappa(m)}\right| \geq 2 \delta
$$

Then select $\nu(m)>\nu(m-1)$ so that

$$
\sum_{n=1+\nu(m-1)}^{\nu(m)}\left|b_{n, \kappa(m)}\right| \geq \delta
$$

and

$$
\sum_{n=\nu(m)}^{\infty}\left|b_{n, \kappa(m)}\right|<t_{m}
$$

Now define $x$ by

$$
x_{k}:=\left\{\begin{array}{cl}
\frac{e^{2 \theta}}{m}, & \text { if } k=\kappa(m), \text { for } m=1,2, \ldots, \text { and } \theta \in \mathbb{R} \\
0, & \text { otherwise. }
\end{array}\right.
$$

This yields $x \in \ell_{A}$ because by (3.7),

$$
\sum_{n=\mu}^{\infty}\left|(A x)_{n}\right| \leq \sum_{n=\mu}^{\infty}\left|\sum_{m=1}^{\infty} a_{n, \kappa(m)}\right|<\sum_{n=\mu}^{\infty} t_{m}
$$

For $B x$, inequalities (3.8), (3.9), and (3.10) give

$$
\begin{aligned}
\sum_{n=1}^{\nu(n)}\left|(B x)_{n}\right| & =\sum_{m=1}^{N} \sum_{n=1+\nu(m-1)}^{\nu(m)}\left|\sum_{j=1}^{\infty} b_{n, \kappa(\jmath)}\left(\frac{1}{j}\right)\right| \\
& \geq \sum_{m=1}^{N} \sum_{n=1+\nu(m-1)}^{\nu(m)}\left\{\left|b_{n, \kappa(m)}\right| \frac{1}{m}-\sum_{\jmath \neq m}\left|b_{n, \kappa(\jmath)}\right|\right\} \\
& =\sum_{m=1}^{N} \sum_{n=1+\nu(m-1)}^{\nu(m)}\left|b_{n, \kappa(m)}\right| \frac{1}{m}-\sum_{m=1}^{N} \sum_{n=1+\nu(m-1)}^{\nu(m)} \sum_{g \neq m}\left|b_{n, \kappa(\jmath)}\right| \\
& \geq \delta \sum_{m=1}^{N} \frac{1}{m}-2 \sum_{\jmath=1}^{\infty} t_{\kappa(\jmath)} .
\end{aligned}
$$

Hence, $B x \notin \ell^{1}$, which establishes the assertion that $x$ is in $\ell_{A}$ but not in $\ell_{B}$.

Note that in defining $x$ we need only have $\left|x_{\kappa(m)}\right| \leq 1 / m$ in order to have the subsequent inequalities valid It is the convergence of the $\mu-1$ series

$$
\sum_{\jmath=1}^{\infty} a_{n, \kappa(\jmath)} x_{\kappa(\jmath)}=(A x)_{n}
$$

for $n=1,2, \ldots, \mu-1$ that requires the factor of $e^{\imath \theta}$ in $x_{\kappa(m)} \quad$ (See Theorem 1 and Lemma 1 of [5])

REMARK. As above with Theorem 1, we have not needed the full strength of the hypotheses, in this case, the assumption that $A$ is an $\ell-\ell$ matrix is stronger than what is needed. Condition $\left(\begin{array}{l}3 \\ 2\end{array}\right)$ guarantees that $A x \in \ell^{1}$ whenever it exists, so the only concern is that $(A x)_{n}$ exists for $n<\mu$ This existence would be guaranteed by assuming only that the row sequences $\left\{a_{n, k(\jmath)}\right\}_{j=1}^{\infty}$ for $n<\mu$ are bounded. (See Lemma 1 of [5].) 
As an illustration of Theorem 3.3, we give an example of two matrices that are noncomparable in the $\ell-\ell$ sense

EXAMPLE 3.1. Define $A$ by

$$
a_{n k}:= \begin{cases}1, & \text { if } n=1 \text { and } k=1,2, . . \\ \frac{1}{n}, & \text { if } k=n>1, \\ 0, & \text { otherwise. }\end{cases}
$$

Take $B$ to be the Euler-Knopp matrix $E_{r}$ for some $r \in(0,1)$.

$$
E_{r}[n, k]:= \begin{cases}\left(\begin{array}{l}
n \\
k
\end{array}\right)(1-r)^{n-k} r^{k}, & \text { if } k \leq n, \\
0, & \text { if } k>n .\end{cases}
$$

Then

$$
\sum_{n=2}^{\infty}\left|a_{n k}\right|=\frac{1}{n}, \quad \text { for } k=2,3, \ldots
$$

so $A$ satisfies condition (3 2). In Theorem 4 of [5], it is noted that for each $k$,

$$
\sum_{n=k}^{\infty}\left|E_{r}[n, k]\right|=\frac{1}{r}
$$

so $E_{\tau}$ does not satisfy (3.2). Hence, by Theorem $3.2, \ell_{A} \nsubseteq \ell_{E_{r}}$. Although the following does not involve Theorems 3.2 and 3.3, for the sake of completeness we show that $\ell_{E_{r}} \nsubseteq \ell_{A}$. This is verified by observing that if $r \in(0,1)$ and $x_{k}:=(-r)^{-k}$, then $\left(E_{r} x\right)_{n}=(-r)^{n}$; therefore $x \in \ell_{E_{r}} \quad$ But $\left|(A x)_{n}\right|=\left|(-r)^{-n} / n\right| \rightarrow \infty$, so $x \notin \ell_{A}$.

In closing we offer an open question related to Theorem 3.2. Can the absolute sums in conditions (3.2) and (3.3) be weakened to ordinary sums? More precisely, if $A$ satisfies

$$
\underset{k}{\liminf }\left|\sum_{n=\mu}^{\infty} a_{n k}\right|=0
$$

and $B$ satisfies

$$
\liminf _{k}\left|\sum_{n=\mu}^{\infty} b_{n k}\right|>0
$$

does it follow that $\ell_{A} \nsubseteq \ell_{B}$ ?

\section{REFERENCES}

[1] AGNEW, R. P., "On equivalence of methods of summation," Tôhoku Math. J. 35 (1932), 244-252

[2] "A simple sufficient condition that a method of summability be stronger than convergence," Bull. Amer. Math. Soc. 52 (1946), 128-132

[3] ,Equivalence of methods for evaluation of sequences," Proc. Amer. Math. Soc. 3 (1952),

[4] FRIDY, J. A., "Mercerian-type theorems for absolute summability," Portugal. Math. 33 (1974), $141-145$

[5] "Absolute summability matrices that are stronger than the identity mapping," Proc. Amer. Math. Soc. 47 (1975), 112-118.

[6] HARDY, G.H, Divergent Series, Clarendon Press, Oxford, 1949

[7] KNOPP, K. and LORENTZ, G. G., "Beitrage zur absoluten Limitierung," Arch. Math. 2 (1949), 10-16.

[8] WILANSKY, A, "An application of Banach linear functionals to summability," Trans. Amer. Math. Soc. 67 (1949), 59-68. 


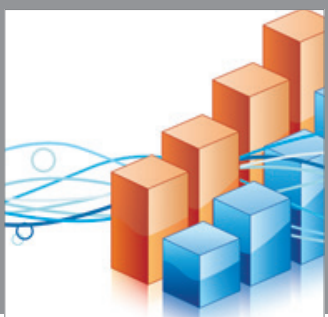

Advances in

Operations Research

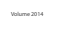

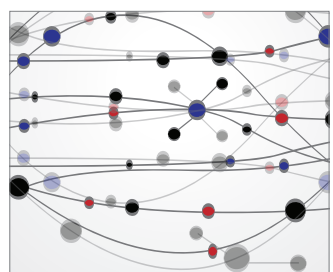

\section{The Scientific} World Journal
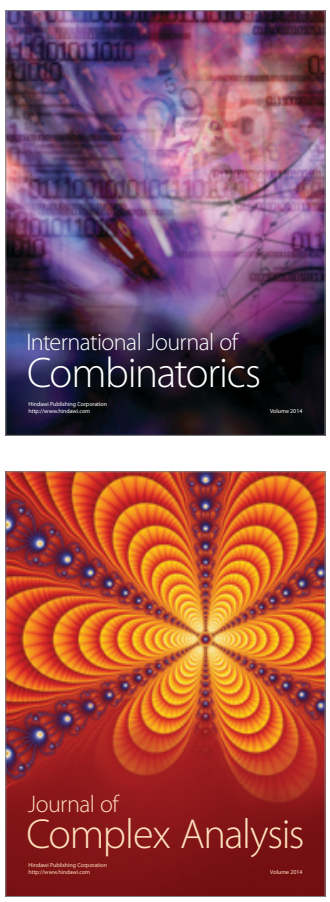

International Journal of

Mathematics and

Mathematical

Sciences
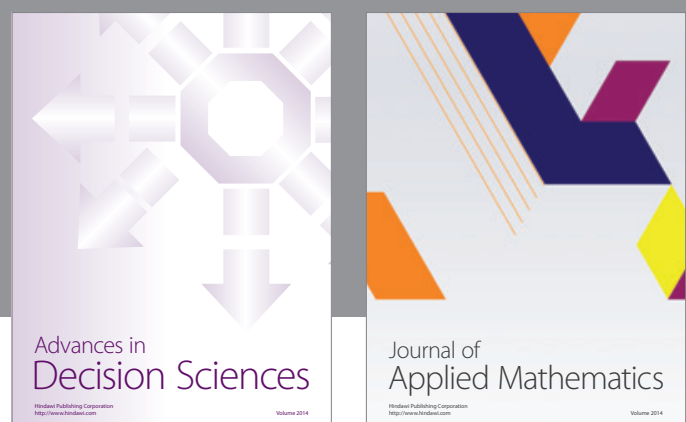

Journal of

Applied Mathematics
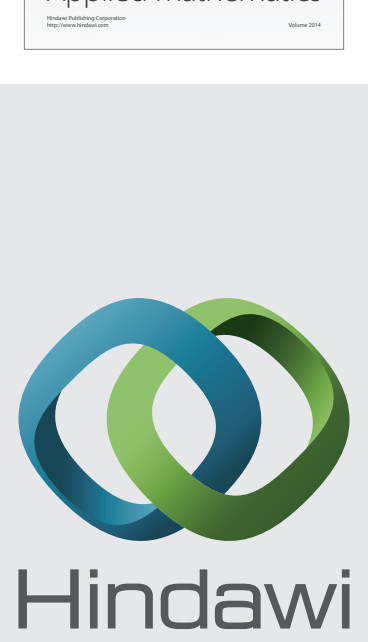

Submit your manuscripts at http://www.hindawi.com
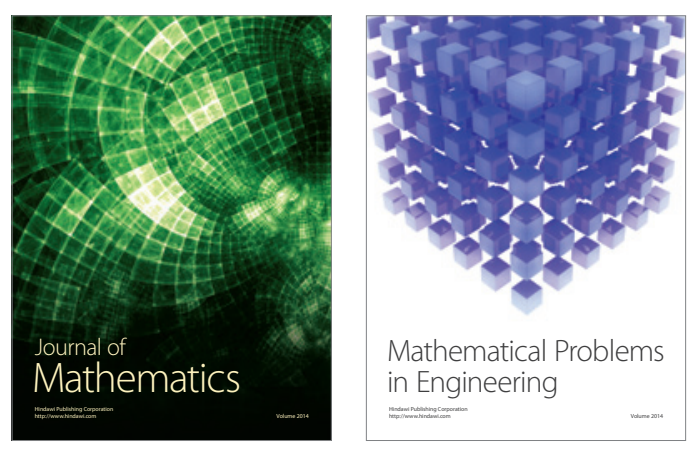

Mathematical Problems in Engineering
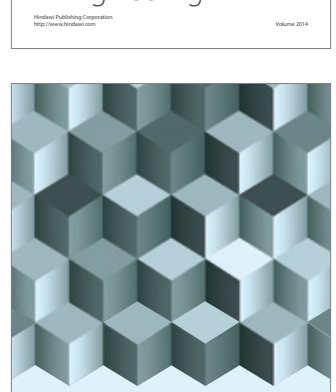

Journal of

Function Spaces
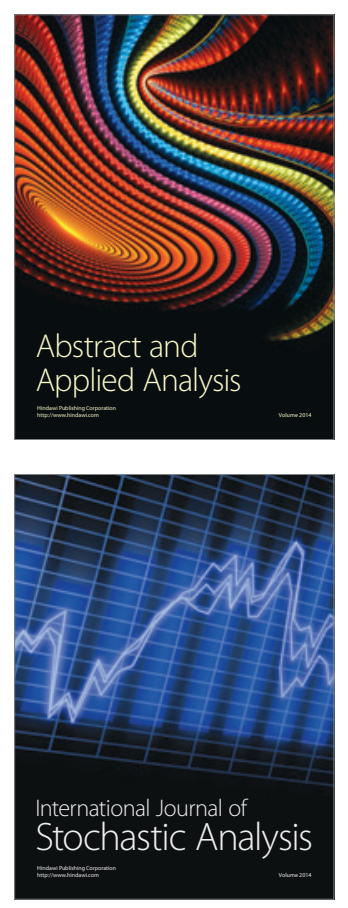

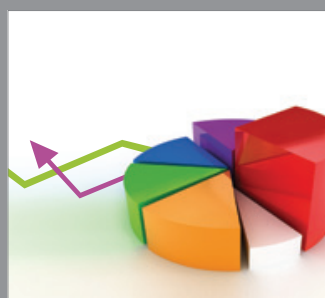

ournal of

Probability and Statistics

Promensencen
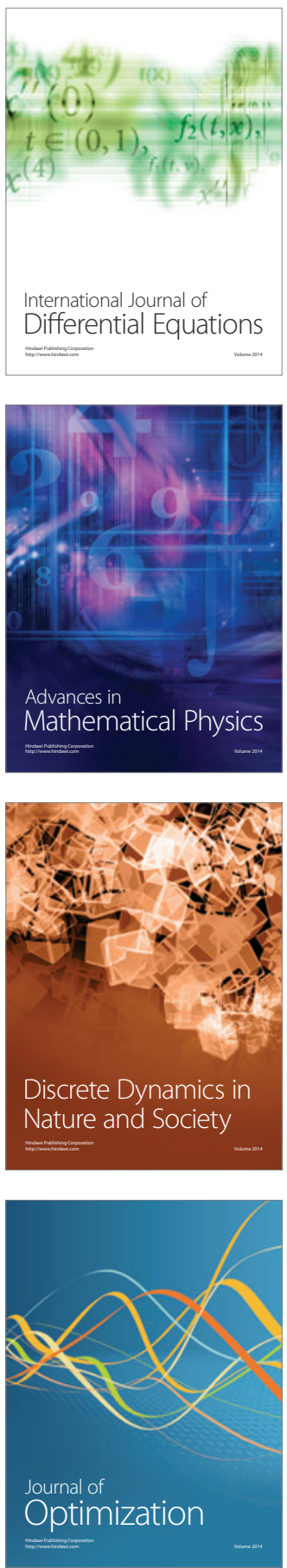